\title{
CircZNF609 promotes breast cancer cell growth, migration, and invasion by elevating P70S6KI via sponging miR-I45-5p
}

This article was published in the following Dove Press journal: Cancer Management and Research

\author{
Shengting Wang' \\ Xukai Xue ${ }^{2}$ \\ Rui Wang' \\ Xiaoming $\mathrm{Li}^{1}$ \\ Qian $\mathrm{Li}^{\prime}$ \\ Yufang Wang' \\ Peijun Xie' \\ Yuhua Kang' \\ Rui Meng' \\ Xinghua Feng' \\ 'Department of Clinical Medicine, \\ Peihua University, Xi'an 710125 , \\ China; ${ }^{2}$ Department of Spine Surgery, \\ Honghui Hospital, Xi'an Jiaotong \\ University, Xi'an 7I0054, China
}

Background: Accumulating evidence suggests that circular RNAs (circRNAs) play critical roles in carcinomas. However, the contributions of circRNAs to breast cancer remain unclear. Herein, we determined the role of circZNF609 in breast cancer.

Methods: A total of 143 breast cancer and 38 normal tissues were collected to assess the expression of circZNF609 and its relationship with breast cancer prognosis. A series of in vitro and in vivo functional experiments were carried out to elucidate the role of circZNF609 in breast cancer progression and its underlying molecular mechanisms.

Results: CircZNF609 was markedly over-expressed in breast cancer tissues and cell lines, and high circZNF609 expression was closely associated with poor outcome. Silencing of circZNF609 inhibited the malignant phenotype of breast cancer in vitro and in vivo. Mechanistically, circZNF609 served as a sponge of miR-145-5p to elevate p70S6K1 expression. Moreover, miR145-5p overexpression or p70S6K1 knockdown abrogated the oncogenic effects of circZNF609 in breast cancer. In addition, clinically, a strong negative correlation was observed between the expression of circZNF609 and miR-145-5p in breast cancer tissues $(r=0.597, P<0.001)$, whereas a positive correlation between circZNF609 and p70S6K1 expression $(\mathrm{r}=0.319, P<0.001)$. Conclusion: These data suggest that circZNF609 contributes to breast cancer progression, at least partly, by modulating the miR-145-5p/p70S6K1 axis, and it may be a potential therapeutic target for breast cancer.

Keywords: CircRNAs, miRNA sponge, breast cancer, progression, prognosis

\section{Introduction}

Breast cancer is considered one of the most common malignancies worldwide. ${ }^{1}$ Despite remarkable advances that have been made in the diagnosis and treatment of breast cancer, the prognosis of high-stage patients is still poor. ${ }^{2}$ Therefore, a better understanding of the regulatory mechanisms underlying breast cancer pathogenesis is urgently needed in the clinical management.

CircRNAs, a novel type of ncRNAs, are characterized by covalent closed-loop structures without $5^{\prime}$-end cup structure and $3^{\prime}$-end poly(A) tail. ${ }^{3}$ Extensive studies show that circRNAs are abundant in eukaryotic cells and play key roles in human diseases, including breast cancer, ${ }^{4,5}$ by serving as the competing endogenous RNAs (ceRNAs). ${ }^{6,7}$ The most famous circRNA is CDR1as, which harbors a large number of miR-7 binding sites. ${ }^{8}$ Subsequently, Zheng et al showed that circHIPK3 could bind to miR-124 to promote cell growth. ${ }^{9}$ Han et al reported that circMTO1 could sponge miR-9 to retard liver cancer progression. ${ }^{10}$ Zhong et al demonstrated that circMYLK promoted bladder cancer angiogenesis and metastasis by interacting
Correspondence: Xinghua Feng Department of Clinical Medicine, Peihua University, 888 Changning Street, Xi'an 710125, Shaanxi, China Emailm18629332769@I63.com 
with endogenous miR-29a. ${ }^{11}$ These above studies reveal that circRNAs participate in cancer development and progression by sponging miRNAs to modulate gene expression. However, the functional interactions between circRNAs and miRNAs on the progression of breast cancer are still not well explored.

It was reported that circZNF609 was indispensable for the growth of central nervous system. ${ }^{12}$ Strikingly, a more recent study found that circZNF609 could be translated into a protein, which was involved in controlling myoblast proliferation. ${ }^{13}$ Meanwhile, circZNF609 was also reported to interact with miR-150-5p to regulate the expression of AKT3 in Hirschsprung's disease. ${ }^{14}$ Lately, Liu et al showed that circZNF609 contributed to vascular endothelial dysfunction by sponging and weakening miR-615-5p activity. ${ }^{15}$ These indicate that circZNF609 may possess various functional roles in human diseases. Nevertheless, its biological role in breast cancer remains unknown.

Here, we investigated the expression of circZNF609 in breast cancer and its potential prognostic value. The functions and mechanisms of circZNF609 in breast cancer progression were also explored.

\section{Materials and methods Clinical specimens}

In this study, we collected 143 breast cancer and 38 adjacent normal tissues from patients who were diagnosed with primary mammary carcinoma and who had undergone surgery in Affiliated Honghui Hospital of Xi' an Jiaotong University (Xi'an, China). Patients who had undergone radiotherapy and chemotherapy were excluded. We got informed consent from each patient, and our study was approved by the ethics committee of Affiliated Honghui Hospital of Xi' an Jiaotong University.

\section{Cell culture and transfection}

All breast cancer cell lines were derived from ATCC (Manassas, VA, USA) and were cultured in DMEM or RPMI1640 and incubated in $5 \% / 37^{\circ} \mathrm{C} \mathrm{CO}_{2}$ atmosphere. si-circZNF609, si-p70S6K1, miR-145-5p inhibitors or mimics, and the respective controls (GenePharma, Shanghai, China) were transfected into cells with Lipofectamine 2000 (Invitrogen, Waltham, MA, USA) according to the manufacturer's protocol. The sequences of si-circZNF609 are as follows:

si-circZNF609\#1:5'-AAGTCTGAAAAGCAATGATGT-3'; si-circZNF609\#2: 5'-GTCAAGTCTGAAAAGCAA TGA-3';

si-circZNF609\#3: 5'-AGTCTGAAAAGCAATGAT GTT-3'.

\section{CircZNF609 plasmid construction and stable transfection}

To construct circZNF609 overexpression plasmids, the human cDNA sequences of circZNF609 were cloned into pcD-ciR vector, which were purchased from Geneseed Biotech Co., Ltd. (Guangzhou, China), followed by transfection with Lipofectamine 2000 (Invitrogen) and selected by G418.

\section{Quantitative reverse transcription-PCR (qRT-PCR)}

We extracted total RNA by TRIzol reagent (Invitrogen). And then the qRT-PCR analysis was performed using SYBR Premix Ex Taq II kit (Takara, Otsu, Japan). The internal standard controls for circRNA/mRNA and miRNA were GAPDH and U6, respectively. The primer sequences are as follows: CircZNF609 (divergent primer): forward: 5'-TGAGTGTCGCCTGCTAAAGA-3'; reverse: 5'-CCCCCAGCTTTCCTATTTTC-3' and ZNF609 (convergent primer): forward: 5'-AATCTTGAGGTGGGACGTTG-3'; reverse: 5'-TCCAGTTTCTGCTGGTCCTT-3'.

\section{Animal studies}

A total of 10 six-week-old female BALB/c nude mice were divided into two groups. Each mouse was subcutaneously injected $5 \times 10^{6}$ MDA-MB-231 cells to establish the mouse xenograft model. On the tenth day, cholesterol-conjugated si-circZNF609 and negative controls (GenePharma) were intratumorally injected. All procedures were approved by the Institutional Animal Care and Use Committee review board of Peihua University, Xi'an, China. And animal experiment operations were in agreement with the Animal Policy and Welfare Committee of Peihua University.

\section{Immunohistochemistry (IHC)}

The method of IHC was previously described. ${ }^{16}$ The H-score value $^{16}$ was used to semiquantify the staining results.

\section{Cell viability assay}

The proliferation of breast cancer cells was assessed by cellcounting kit (CCK)-8 assay. MCF7 and MDA-MB-231 cells were plated into the 96-well plates, followed by incubation with $10 \mu \mathrm{L}$ of CCK-8 reagent for 2 hours. The absorbance values at $450 \mathrm{~nm}$ were tested by using a microplate reader (Tecan, Männedorf, Switzerland).

\section{Ethynyldeoxyuridine (EdU) incorporation assay}

The EdU kit was purchased from RiboBio Company (Guangzhou, China); and $50 \mathrm{mM}$ EdU reagent was added into 
96-well plates with the transfected cells and incubated for 4 hours, followed by fixing and staining. Images were obtained with a fluorescence microscope.

\section{Transwell migration and invasion assays}

The 24-well transwell chamber, without or with Matrigel (Corning, NY, USA), was utilized to determine breast cancer cell migration/invasion. After 24 hours of incubation, the migrated or invaded cells were fixed and stained, subsequently analyzed under a microscope.

\section{Wound healing assay}

Cells were cultured in six-well plates and scraped with a sterile $200 \mu \mathrm{L}$ pipette tip. Photographic images were taken at the indicated time and subsequently analyzed using Image $\mathrm{J}$ software.

\section{Dual-Luciferase reporter assay}

The wild-type or mutated sequences of circZNF609 and p70S6K1 3'-untranslated region (3'-UTR) were cloned into pmirGLO vector. Cells were, respectively, cotransfected with these reporter plasmids and miR-145-5p inhibitors or mimics. After 48 hours, the luciferase activity was tested by a DualLuciferase assay system (Promega Corporation, Fitchburg, WI, USA) according to the manufacturer's protocol.

\section{Western blotting}

Breast cancer cells were lysed and harvested with $200 \mu \mathrm{L}$ RIPA lysis buffer, followed by electrophoresis using $10 \%$ SDS-PAGE. Then incubated with anti-p70S6K1 antibody (\#14485-1-AP, Proteintech, Chicago, IL, USA) and antiGAPDH antibody (\#ab181602, Abcam, Cambridge, UK) at $4^{\circ} \mathrm{C}$ overnight and subsequently incubated with secondary antibodies. The signals were tested using a chemiluminescence system (Bio-Rad Laboratories Inc., Hercules, CA, USA).

\section{Statistical analysis}

Student's $t$-test was used to evaluate the differences between the different groups. Kaplan-Meier plot was utilized to analyze the survival curves. Correlations were measured by the Pearson correlation analysis. $P<0.05$ was considered statistically significant.

\section{Results \\ CircZNF609 is significantly overexpressed and predicts poor prognosis in breast cancer}

To investigate the potential role of circZNF609 in breast cancer, we measured the expression pattern of circZNF609 in breast cancer tissues and cell lines. CircZNF609 was markedly overexpressed in breast cancer tissues $(n=143)$ compared with adjacent normal tissues $(n=38)$ (Figure 1A). Consistent with our findings in breast cancer tissues, we found that circZNF609 expression levels are unanimously increased in all breast cancer cell lines (Figure 1B). More importantly, high circZNF609 expression was significantly related to lymph node metastasis $(P<0.001)$, Ki-67 proliferation index $(P=0.024)$, advanced TNM stage $(P=0.006)$, and poor overall survival ( $P=0.002$ ) (Table 1) (Figure 1C). These data suggest its potential oncogenic role in breast cancer.

\section{Silencing of circZNF609 inhibits breast cancer growth in vivo}

To further examine the oncogenic activity of circZNF609 in breast cancer, we established xenograft tumor model by subcutaneously injecting MDA-MB-231 cells $(n=5$ for each group). Tumor volume was measured every 3-4 days with digital calipers. When the tumor volume reached about $100 \mathrm{~mm}^{3}$, cholesterol-conjugated si-circZNF609 and negative controls were injected intratumorally. Twentyeight days later, mice were euthanized. The results showed that circZNF609 knockdown suppressed the tumor growth rate and tumor volume (Figure 1D-F). IHC for Ki-67 also confirmed these results (Figure $1 \mathrm{G}, \mathrm{H}$ ). These data reveal that inhibition of circZNF609 can effectively retard breast cancer progression.

\section{Knockdown of circZNF609 suppresses the capability of breast cancer cells to proliferate, migrate, and invade}

We next interrogated whether circZNF609 could regulate biological activity in breast cancer. Three siRNAs targeting circZNF609 were designed and synthesized and subsequently transfected into MCF7 and MDA-MB-231 cells These siRNAs effectively decreased circZNF609 expression, while they had no effect on its liner isoform (Figure 2A). si-circZNF609\#1 exerted the highest inhibitory efficiency (Figure 2A) and was selected for the subsequent experiments. The CCK-8 and EdU incorporation assays showed that circZNF609 silencing obviously inhibited MCF7 and MDA-MB-231 cell proliferation (Figure 2B, C). Moreover, transwell migration and invasion assays showed that depletion of circZNF609 retarded breast cancer cell migration and invasion (Figure 2D). These above findings demonstrate that knockdown of circZNF609 suppresses the malignant phenotypes of breast cancer cells. 

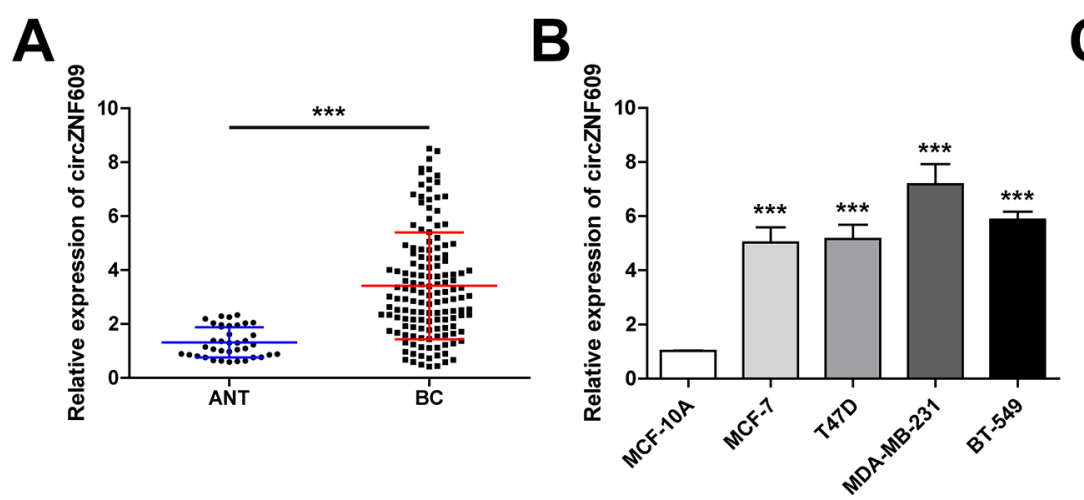

C

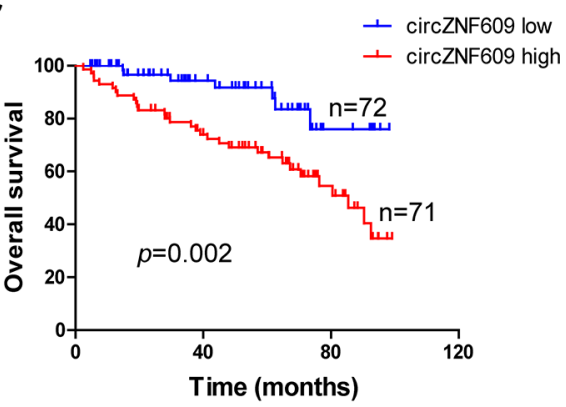

D
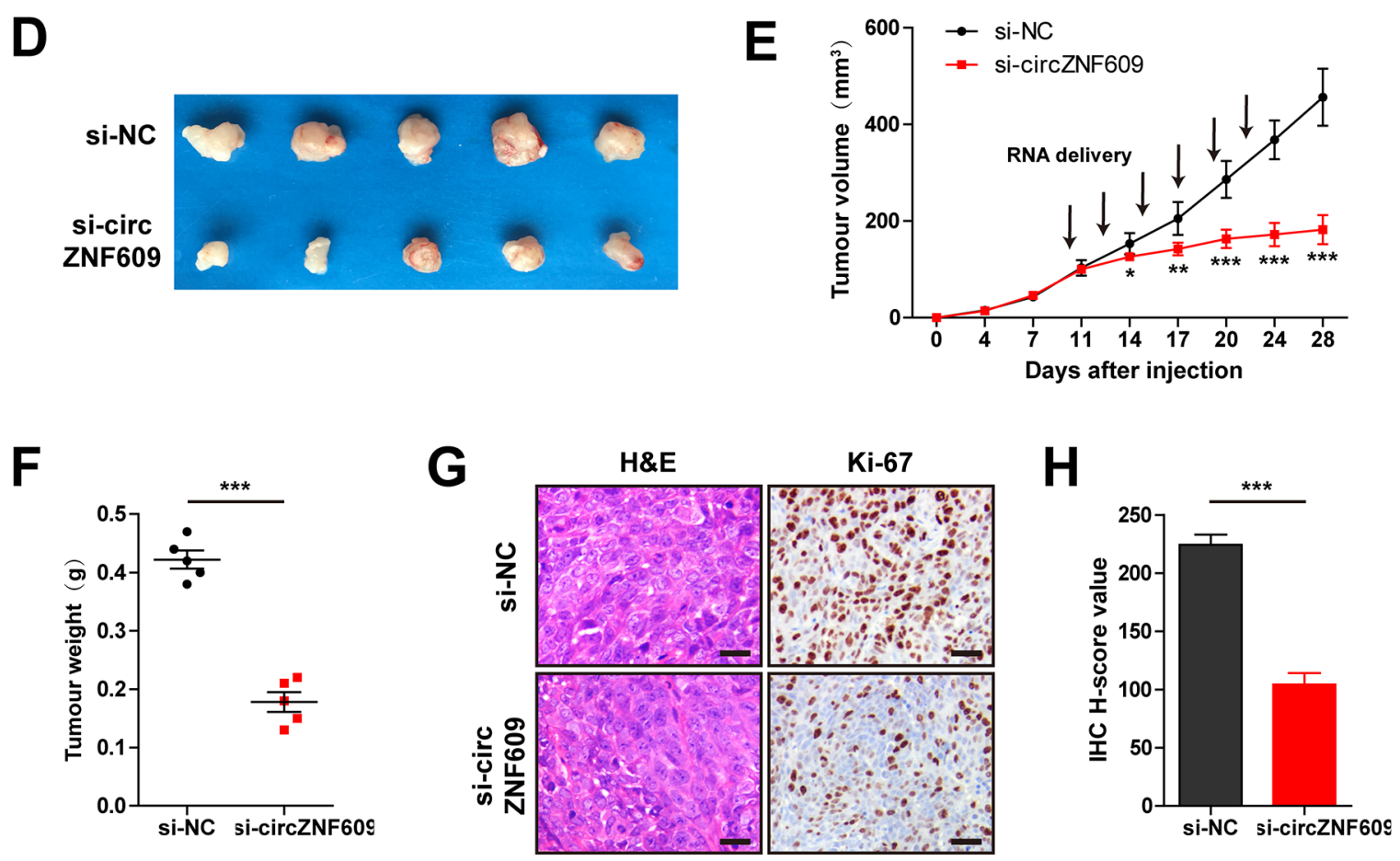

Figure I The role of circZNF609 in breast cancer.

Notes: (A) qRT-PCR analysis of circZNF609 in BC tissues ( $n=143$ ) and adjacent normal tissues ( $n=38$ ). (B) qRT-PCR analysis of circZNF609 in BC cell lines. (C) KaplanMeier plot of $B C$ patients with low $(n=72)$ or high $(n=71)$ circZNF609 expression. Using median circZNF609 values as cutoff. (D) The images of subcutaneous tumor from two treatment groups $(n=5)$. (E) Tumor volumes of nude mice were measured every 3-4 days. (F) Tumor weights of nude mice were measured on the 28th day. (G) Representative images of H\&E staining for tissue morphology and IHC staining for proliferation (Ki-67). Scale bar=20 $\mu \mathrm{m}$. (H) The statistical plots for Ki67 H-score in two treatment groups. $* P<0.05, * * P<0.01$, $* * * P<0.001$

Abbreviations: ANT, adjacent normal tissue; BC, breast cancer; NC, negative control; IHC, immunohistochemistry; qRT-PCR, quantitative reverse transcription-PCR.

\section{CircZNF609 serves as a sponge of miR- I45-5p}

Given that circRNAs were proposed to function as sponges for miRNAs, ${ }^{17}$ we tried to assess whether circZNF609 could bind to miRNAs. By searching the web tool named CircInteractome, we speculated that circZNF609 may have a putative binding site with miR-145-5p (Figure 3A), which is a well-known tumor suppressor. To verify this hypothesis, we conducted luciferase reporter and RNA pull-down assays. The reported plasmids were cotransfected with miR-145-5p mimics or negative controls into MCF7 and MDA-MB-231 cells. As shown in Figure 3B, miR-145-5p overexpression decreased the luciferase activity of the wild-type vector by at least $60 \%$, but did not change the mutated one. And RNA pull-down results revealed that circZNF609 did interact with miR-145-5p (Figure 3C). Furthermore, knockdown of circZNF609 increased, but overexpression of circZNF609 decreased miR-145-5p expression (Figure 3D). Analogously, miR-145-5p overexpression reduced, whereas miR-145-5p silencing elevated circZNF609 expression in these two 
Table I Relationship between circZNF609 expression and clinicopathological features of breast cancer patients $(n=143)$

\begin{tabular}{|c|c|c|c|c|}
\hline \multirow[t]{2}{*}{ Parameters } & \multirow[t]{2}{*}{ Total $(n=143)$} & \multicolumn{2}{|c|}{ circZNF609 expression ${ }^{a}$} & \multirow[t]{2}{*}{$P$-value } \\
\hline & & Low $(n=72)$ & High $(n=7 I)$ & \\
\hline \multicolumn{5}{|l|}{ Age (years) } \\
\hline$\leq 40$ & 35 & 14 & 21 & 0.177 \\
\hline$>40$ & 108 & 58 & 50 & \\
\hline \multicolumn{5}{|c|}{ Tumor size $(\mathrm{cm})$} \\
\hline$\leq 2$ & 83 & 39 & 44 & 0.344 \\
\hline$>2$ & 60 & 33 & 27 & \\
\hline \multicolumn{5}{|l|}{ LN metastasis } \\
\hline Negative & 89 & 58 & 31 & $<0.001$ \\
\hline Positive & 54 & 14 & 40 & \\
\hline \multicolumn{5}{|l|}{ TNM stage } \\
\hline I-II & 67 & 42 & 25 & 0.006 \\
\hline III-IV & 76 & 30 & 46 & \\
\hline \multicolumn{5}{|l|}{ ER status } \\
\hline Negative & 40 & 18 & 22 & 0.425 \\
\hline Positive & 103 & 54 & 49 & \\
\hline \multicolumn{5}{|l|}{ PR status } \\
\hline Negative & 44 & 24 & 20 & 0.503 \\
\hline Positive & 99 & 48 & 51 & \\
\hline \multicolumn{5}{|l|}{ HER2 status } \\
\hline Negative & 111 & 55 & 56 & 0.722 \\
\hline Positive & 32 & 17 & 15 & \\
\hline \multicolumn{5}{|l|}{ Ki-67 status } \\
\hline$\leq 14 \%$ & 36 & 24 & 12 & 0.024 \\
\hline$>14 \%$ & 107 & 48 & 59 & \\
\hline
\end{tabular}

Note: Using median circZNF609 value as cutoff. Bold values indicate $P<0.05$.

Abbreviations: ER, estrogen receptor; LN, lymph node; PR, progesterone receptor.

cells (Figure 3E). In addition, miR-145-5p was markedly decreased in breast cancer tissues when compared with matched normal tissues (Figure 3F). And a strong negative correlation was observed between the expression of circZNF609 and miR-145-5p in breast cancer tissues $(r=-0.597$, $P<0.001$ ) (Figure 3G). Altogether, these results suggest that circZNF609 may exert its biological function by sponging miR-145-5p.

\section{CircZNF609 sponges and sequesters miR- I45-5p to upregulate $\mathrm{p} 70 \mathrm{S6} \mathrm{K}$ I expression}

It has been reported that $\mathrm{p} 70 \mathrm{~S} 6 \mathrm{~K} 1$ was a target of miR$145-5 p .{ }^{18}$ To confirm this result, we carried out luciferase reporter assay and found that miR-145-5p overexpression reduced the luciferase activity of the wild-type vector (Figure 4A), but not the mutated one (Figure 4B). We then cotransfected the luciferase reporter of wild-type p70S6K1 3'-UTR with circZNF609, the results showed that the luciferase activity was markedly increased compared with the control group (Figure 4C). However, this effect was abolished by overexpression of miR-145-5p (Figure 4C), suggesting that
circZNF609 modulated the expression of p70S6K1 via binding to miR-145-5p. Moreover, we found that circZNF609 expression was positively correlated with $\mathrm{p} 70 \mathrm{~S} 6 \mathrm{~K} 1$ expression in breast cancer tissues $(r=0.319, P<0.001)$ (Figure 4D). And the mRNA and protein expression levels of p70S6K1 were obviously rescued in miR-145-5p-overexpressing MCF7 and MDA-MB-231 cells after ectopic expression of circZNF609 (Figure 4E, F). These data show that circZNF609 elevates oncogene $\mathrm{p} 70 \mathrm{~S} 6 \mathrm{~K} 1$ expression by acting as a sponge of miR-145-5p in breast cancer.

\section{miR-I45-5p overexpression or p70S6KI silencing effectively reverses circZNF609- induced promotion of breast cancer progression}

To further confirm whether circZNF609 regulates breast cancer progression through an miR-145-5p/p70S6K1 axis, circZNF609 expression vector and miR-145-5p mimics were cotransfected into MCF7 cells. Ectopic expression of circZNF609 resulted in increased EdU-positive breast cancer cell population, but this proliferation-promoting 

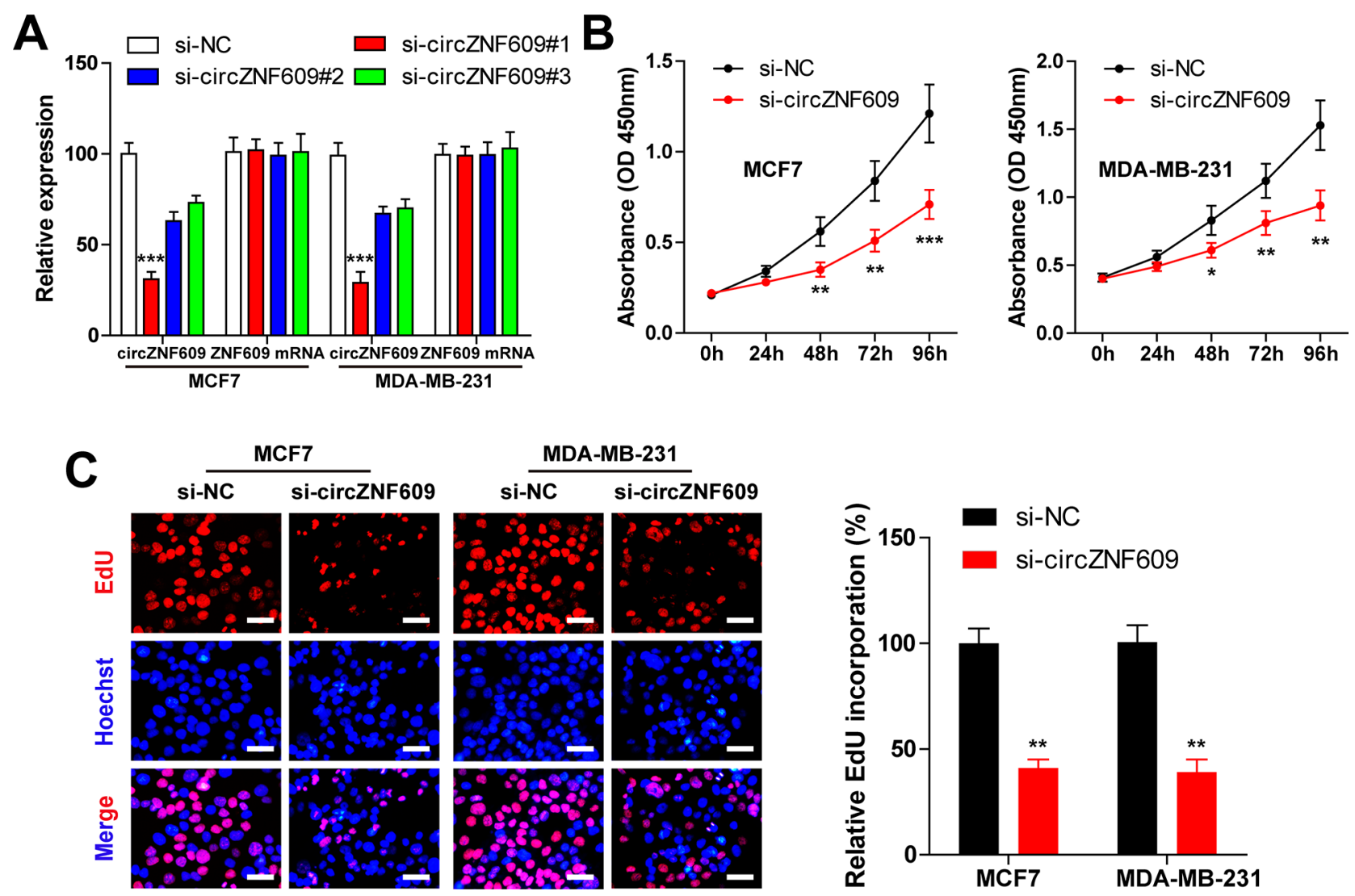

D
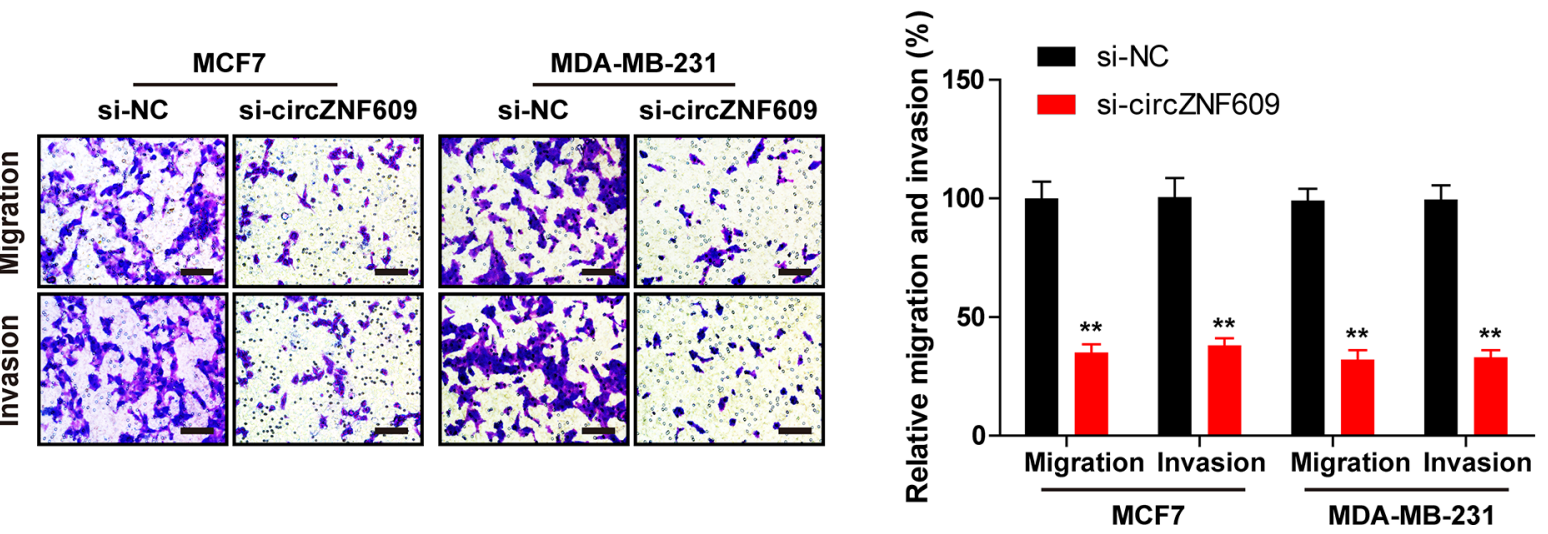

Figure 2 Knockdown of circZNF609 inhibits breast cancer cell proliferation, migration, and invasion in vitro.

Notes: (A) qRT-PCR analysis of the interfering efficacies of three circZNF609-targeting siRNAs on circZNF609 and ZNF609 in MCF7 and MDA-MB-23I cell lines. (B) CCK-8 assay of MCF7 and MDA-MB-23I cells transfected with si-circZNF609\#I or NC. (C) EdU assay of MCF7 and MDA-MB-23I cells transfected with si-circZNF609\# I or NC. Scale bar=20 $\mu \mathrm{m}$. (D) Transwell migration and Matrigel invasion assay of MCF7 and MDA-MB-23I cells transfected with si-circZNF609\#I or NC. Scale bar=20 $\mu \mathrm{m}$. Data were represented as mean $\pm S D$ of at least three independent experiments. $* P<0.05, * * P<0.01$, $* * * P<0.00$ I.

Abbreviations: EdU, ethynyldeoxyuridine; NC, negative control; qRT-PCR, quantitative reverse transcription-PCR; CCK-8, cell-counting kit-8.

effect could be obviously abrogated by miR-145-5p overexpression (Figure 5A). Similar results were also observed in transwell migration and invasion assays without or with Matrigel, in which miR-145-5p overexpression significantly reversed the circZNF609-induced increase in cell migration and invasion rates (Figure 5B), which was in accordance with the wound healing assay (Figure 5C). Of note, circZNF609 cotransfected with si-p70S6K1 mimicked the effect of cotransfected with miR-145-5p mimics on breast cancer malignant phenotypes (Figure $5 \mathrm{~A}-\mathrm{C})$. Taken together, these results demonstrate that miR-145-5p overexpression or p70S6K1 silencing can 


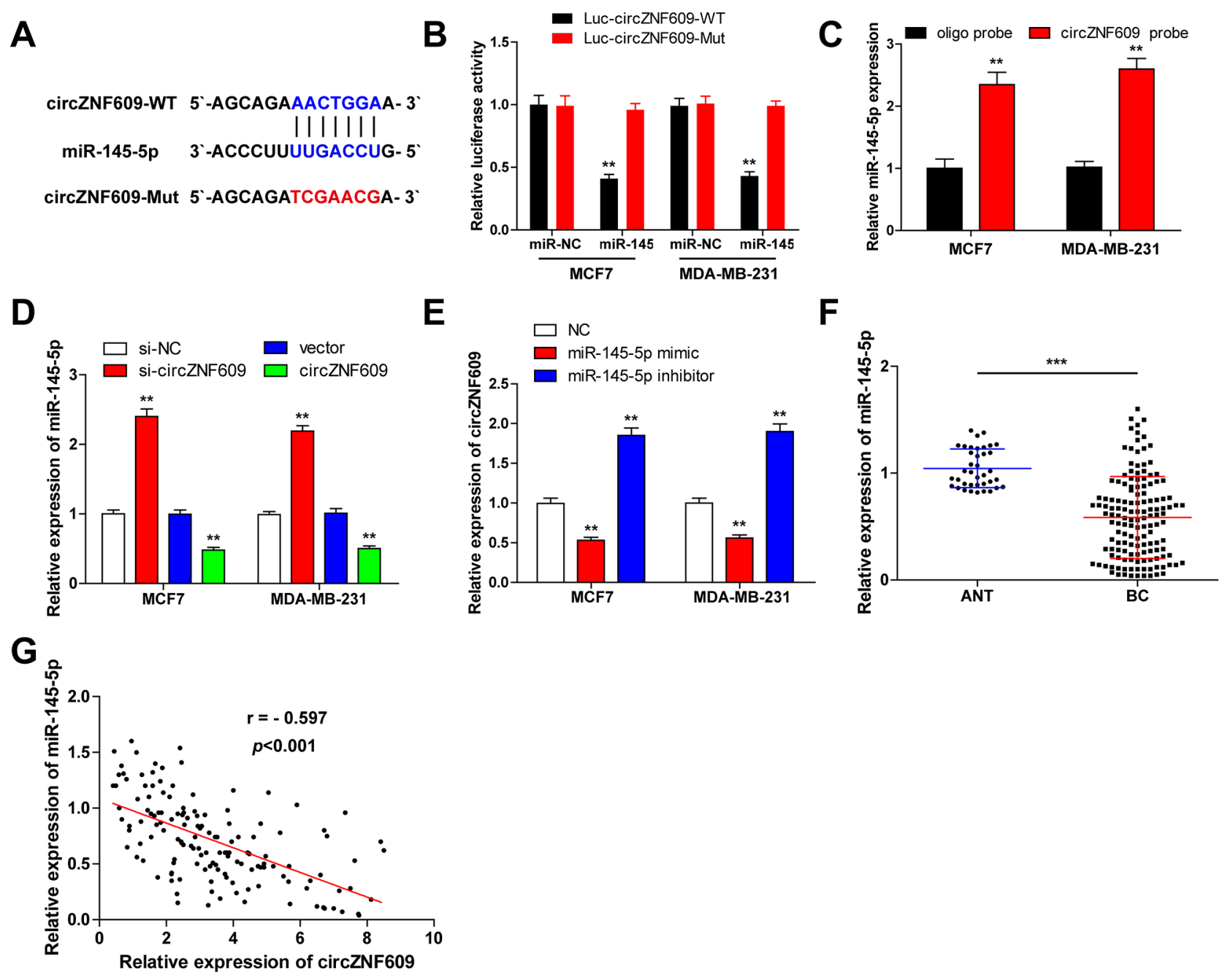

Figure 3 CircZNF609 serves as a sponge of miR-145-5p in breast cell lines.

Notes: (A) The putative targeting site of circZNF609 and miR-145-5p was predicted by Circlnteractome. (B) Luciferase activity analysis in MCF7 and MDA-MB-23I cells cotransfected with miR-I45-5p mimics and pmirGLO-circZNF609-WT or pmirGLO-circZNF609-Mut vector. (C) RNA pull-down assay in MCF7 and MDA-MB-23I cells, the expression levels of miR-145-5p pulled down by circZNF609 or oligo probe were detected by qRT-PCR. (D) qRT-PCR analysis of miR-I45-5p in MCF7 and MDAMB-23I cells with circZNF609 knockdown or overexpression. (E) qRT-PCR analysis of circZNF609 in MCF7 and MDA-MB-23I cells with miR-I45-5P knockdown or overexpression. (F) qRT-PCR analysis of miR- I45-5p in BC tissues ( $n=143$ ) and adjacent normal tissues ( $n=38$ ). (G) Pearson's correlation analysis of circZNF609 and miR145-5p in $B C$ tissues $(n=\mid 43)(r=-0.597, P<0.00 I)$. Data were represented as mean $\pm S D$ of at least three independent experiments. $* * P<0.01$, $* * * P<0.00 I$. Abbreviations: ANT, adjacent normal tissue; BC, breast cancer; NC, negative control WT, wild type; qRT-PCR, quantitative reverse transcription PCR.

reverse circZNF609-induced aggressive phenotypes of breast cancer cells.

\section{Discussion}

Nowadays, the study of circRNA is receiving more and more attention. ${ }^{19}$ Here, we found that circZNF609 was remarkably upregulated in breast cancer tissues and was closely related to poor prognosis. Furthermore, circZNF609 promoted breast cancer aggressive phenotypes by functioning as a sponge of miR-145-5p, thereby elevating oncogene p70S6K1 expression. Thus, the regulatory network involving circZNF609/miR-145-5p/p70S6K1 axis may highlight a better understanding of breast tumorigenesis and development.

Wealth of studies shows that circRNAs are not the byproducts in the process of splicing but a class of noncoding RNA with various biological functions that serve as ceRNAs. ${ }^{5,20}$ For instance, circHIPK 3 could, respectively, sponge miR-30a, miR-7, miR-124, and miR-558 in diabetic proliferative retinopathy, liver cancer, and bladder cancer., ${ }^{9,21}$ CircRNA_100290 could sponge miR-29 family in oral cancer, circLMO7 could sponge miR-378a-3p in myoblasts, ${ }^{24}$ and so on. Herein, we found that circZNF609 functioned as the sponge of miR-145-5p and promoted breast cancer. One 
A

P70S6K1 3'-UTR WT 5'-AAGCUUAACUGGAA- 3'

|| || ||

miR-145-5p

3`-ACCCUUUUGACCUG- 5

p70S6K1 3'-UTR Mut 5'-AAGCUUGCAAUCGA- $3^{\prime}$

C

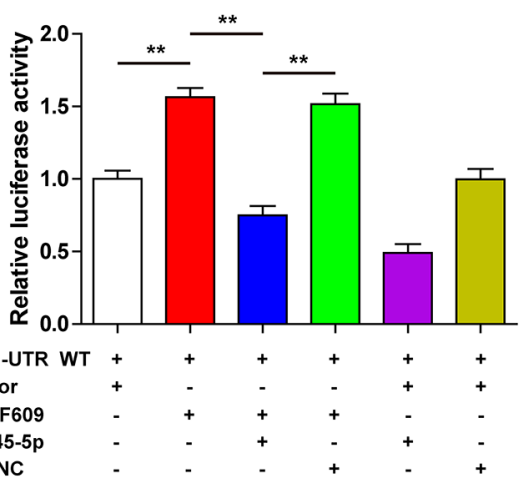

E

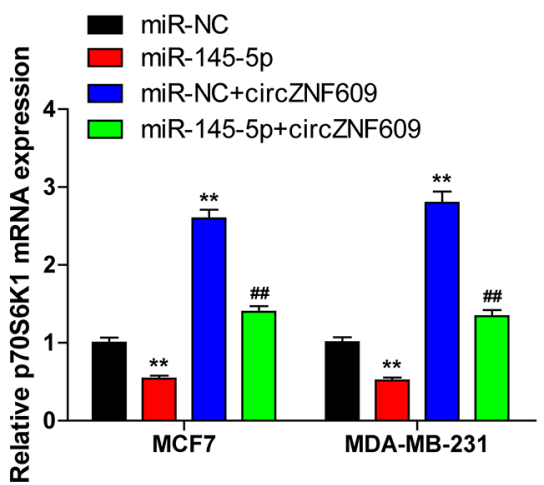

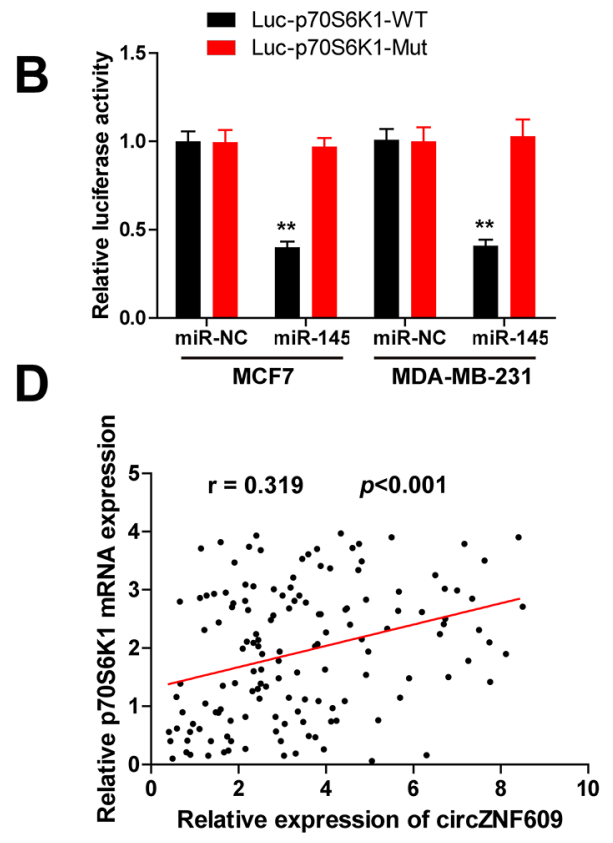

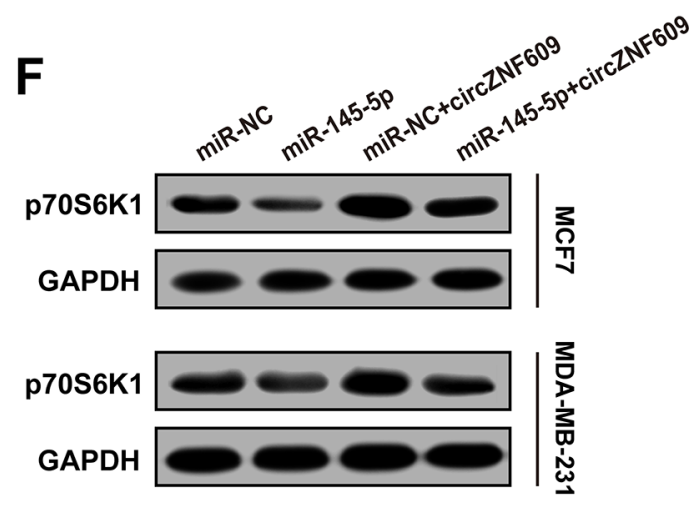

Figure 4 CircZNF609 upregulates p70S6KI expression by sponging miR-145-5p.

Notes: (A) The putative binding site of miR-145-5p and p70S6K 3'-UTR. (B) Luciferase activity analysis in MCF7 and MDA-MB-23I cells cotransfected with miR-I45-5p mimics and pmirGLO-p70S6KI 3'-UTR-WT or pmirGLO-p70S6KI 3'-UTR-Mut vector. (C) Luciferase activity analysis in MCF7 cells cotransfected with miR-145-5p mimics or miR-NC, circZNF609 vector or control vector, and pmirGLO-p70S6KI 3'-UTR-WT vector. (D) Pearson's correlation analysis of circZNF609 and p70S6KI in breast cancer tissues $(n=143)(r=0.319, P<0.001)$. (E) qRT-PCR analysis of p70S6KI mRNA expression in MCF7 and MDA-MB-23I cells cotransfected with miR-I45-5p mimics or miR-NC, and circZNF609 vector or control vector. (F) Western blot analysis of p70S6KI protein expression in MCF7 and MDA-MB-23I cells cotransfected with miR-I45$5 p$ mimics or miR-NC, and circZNF609 vector or control vector. Data were represented as mean \pm SD of at least three independent experiments. * vs control group, ${ }^{*}$ vs miR-145-5p mimics group. ${ }^{*} * P<0.01,{ }^{\#} P<0.01$.

Abbreviations: NC, negative control; UTR, unsaturated region; WT, wild type; qRT-PCR, quantitative reverse transcription PCR.

recent research suggested that circZNF609 could sponge miR-150-5p to regulate AKT3 expression in Hirschsprung's disease. ${ }^{14}$ However, we did not detect if circZNF609 could interact with miR-150-5p in breast cancer cells (data not shown). We speculate that circZNF609 may exert its biological roles in tissue- or cell-specific context. Therefore, the molecular mechanism and function of circZNF609 still require further investigation in other malignancies.

A large number of studies indicate that miRNAs are involved in many important physiological processes..$^{25,26}$ miR-145-5p, a famous tumor suppressor, was frequently downregulated in esophageal squamous cell carcinoma, ${ }^{27}$ prostate cancer, ${ }^{28}$ non-small cell lung cancer, ${ }^{29}$ and colorectal cancer. ${ }^{30}$ Some studies have shown that miR-145-5p inhibited tumor angiogenesis and growth by directly binding to p70S6K1 $3{ }^{\prime}$-UTR. ${ }^{18}$ Our data also confirmed their interaction with breast cancer cells by Dual-Luciferase reporter assay. p70S6K1, a downstream AKT/mTOR signaling pathway, has been reported as a key regulator of cell growth, metabolism, survival, and drug resistance. ${ }^{31}$ p70S6K1 was markedly overexpressed in many human cancers and was closely related to tumor malignancy and poor prognosis. ${ }^{32,33}$ In this study, 


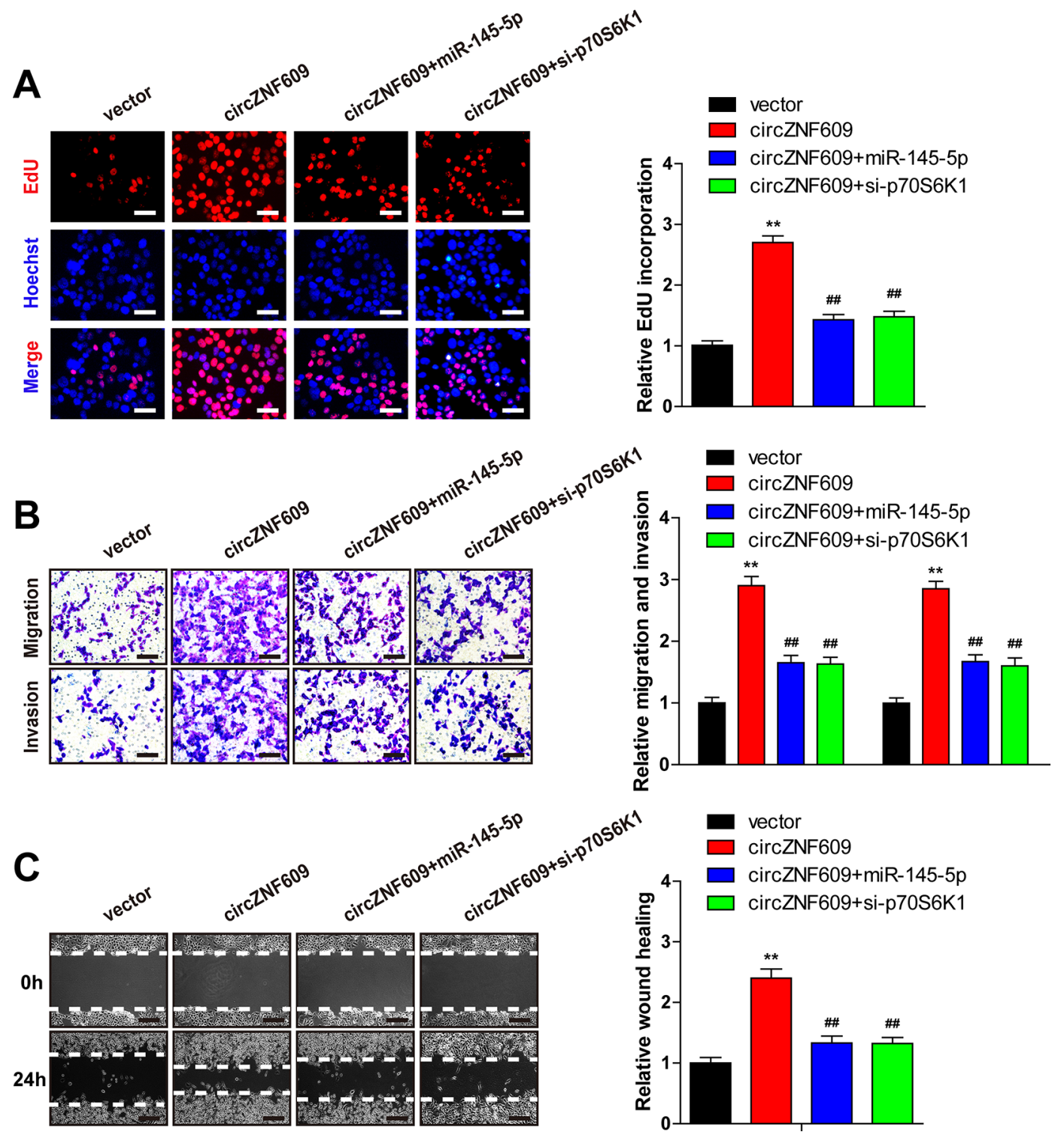

Figure 5 miR-145-5p overexpression or p70S6KI silencing effectively reverses circZNF609-induced promotion of breast cancer progression.

Notes: (A) EdU assay of MCF7 cells cotransfected with circZNF609 vector or control vector, and miR-145-5p mimics or si-p70S6KI. Scale bar=20 $\mu \mathrm{m}$. (B, C) Transwell and wound healing assays of MCF7 cells cotransfected with circZNF609 vector or control vector, and miR-145-5p mimics or si-p70S6KI. Scale bar=20 $\mu \mathrm{m}$. The corresponding statistical plots were exhibited in right panel. Data were represented as mean \pm SD of at least three independent experiments. * vs control vector group, \# vs circZNF609 vector group. $* * P<0.01, \ldots P<0.01$.

Abbreviation: EdU, ethynyldeoxyuridine.

we found that miR-145-5p could significantly suppress the mRNA and protein expression of p70S6K1 and that the inhibition was retarded after ectopic expression of circZNF609, suggesting that circZNF609 increased oncogene p70S6K1 expression by sponging and sequestering miR-145-5p activity. Moreover, ectopic expression of miR-145-5p or silencing of p70S6K1 could effectively reverse circZNF609-induced promotion of breast cancer proliferation, migration, and invasion. Besides, we also found that circZNF609 was negatively and positively correlated with miR-145-5p and p70S6K1 in breast cancer tissues, respectively. These results showed the circZNF609/miR-145-5p/p70S6K1 axis is involved in the pathogenesis of breast cancer.

To sum up, our findings reveal that circZNF609 plays an oncogenic role in breast cancer, at least partly, by elevating p70S6K1 expression via sponging miR-145-5p, and it can also be a promising prognostic biomarker for patient with breast cancer. Our data also demonstrate that targeting the circZNF609/miR-145-5p/p70S6K1 axis may be a novel potential therapeutic strategy for breast cancer. 


\section{Acknowledgment}

This project was supported by grants from High-End Talent Development Fund of Peihua University.

\section{Disclosure}

The authors report no conflicts of interest in this work.

\section{References}

1. Desantis CE, Ma J, Goding Sauer A, Newman LA, Jemal A. Breast cancer statistics, 2017, racial disparity in mortality by state. CA Cancer J Clin. 2017;67(6):439-448.

2. Harjes U. Breast cancer: staying silent. Nat Rev Cancer. 2018;18(3):136.

3. Hentze MW, Preiss T. Circular RNAs: splicing's enigma variations. Embo J. 2013;32(7):923-925.

4. Nair AA, Niu N, Tang X, et al. Circular RNAs and their associations with breast cancer subtypes. Oncotarget. 2016;7(49):80967-80979.

5. Kristensen LS, Hansen TB, Venø MT, Kjems J. Circular RNAs in cancer: opportunities and challenges in the field. Oncogene. 2018;37(5):555-565.

6. Han B, Chao J, Yao H. Circular RNA and its mechanisms in disease: from the bench to the clinic. Pharmacol Ther. 2018;187:31-44.

7. Dong Y, He D, Peng Z, et al. Circular RNAs in cancer: an emerging key player. J Hematol Oncol. 2017;10(1):2.

8. Memczak S, Jens M, Elefsinioti A, et al. Circular RNAs are a large class of animal RNAs with regulatory potency. Nature. 2013;495(7441):333-338.

9. Zheng Q, Bao C, Guo W, et al. Circular RNA profiling reveals an abundant circHIPK3 that regulates cell growth by sponging multiple miRNAs. Nat Commun. 2016;7:11215.

10. Han D, Li J, Wang H, et al. Circular RNA circMTO1 acts as the sponge of microRNA-9 to suppress hepatocellular carcinoma progression. Hepatology. 2017;66(4):1151-1164.

11. Zhong Z, Huang M, Lv M, et al. Circular RNA MYLK as a competing endogenous RNA promotes bladder cancer progression through modulating VEGFA/VEGFR2 signaling pathway. Cancer Lett. 2017;403:305-317.

12. Rybak-Wolf A, Stottmeister C, Glažar P, et al. Circular RNAs in the mammalian brain are highly abundant, conserved, and dynamically expressed. Mol Cell. 2015;58(5):870-885.

13. Legnini I, di Timoteo G, Rossi F, et al. Circ-ZNF609 is a circular RNA that can be translated and functions in myogenesis. Mol Cell. 2017;66(1):22-37.

14. Peng L, Chen G, Zhu Z, et al. Circular RNA ZNF609 functions as a competitive endogenous RNA to regulate AKT3 expression by sponging miR-150-5p in Hirschsprung's disease. Oncotarget. 2017;8(1):808-818.

15. Liu C, Yao MD, Li CP, et al. Silencing of circular RNA-ZNF609 ameliorates vascular endothelial dysfunction. Theranostics. 2017;7(11):2863-2877.
16. Zeng K, Wang Z, Ohshima K, et al. BRAF V600E mutation correlates with suppressive tumor immune microenvironment and reduced disease-free survival in Langerhans cell histiocytosis. Oncoimmunology. 2016;5(7):e1185582.

17. Hansen TB, Jensen TI, Clausen BH, et al. Natural RNA circles function as efficient microRNA sponges. Nature. 2013;495(7441):384-388.

18. Xu Q, Liu LZ, Qian X, et al. MiR-145 directly targets p70S6K1 in cancer cells to inhibit tumor growth and angiogenesis. Nucleic Acids Res. 2012;40(2):761-774.

19. Croce CM. Genetics: are circRNAs involved in cancer pathogenesis? Nat Rev Clin Oncol. 2016;13(11):658.

20. Thomson DW, Dinger ME. Endogenous microRNA sponges: evidence and controversy. Nat Rev Genet. 2016;17(5):272-283.

21. Shan K, Liu C, Liu BH, et al. Circular noncoding RNA HIPK3 mediates retinal vascular dysfunction in diabetes mellitus. Circulation. 2017;136(17):1629-1642.

22. Li Y, Zheng F, Xiao X, et al. CircHIPK3 sponges miR-558 to suppress heparanase expression in bladder cancer cells. EMBO Rep. 2017;18(9):1646-1659.

23. Zeng $\mathrm{K}$, Chen $\mathrm{X}, \mathrm{Xu} \mathrm{M}$, et al. CircHIPK3 promotes colorectal cancer growth and metastasis by sponging miR-7. Cell Death Dis. 2018;9(4):417.

24. Wei X, Li H, Yang J, et al. Circular RNA profiling reveals an abundant circLMO7 that regulates myoblasts differentiation and survival by sponging miR-378a-3p. Cell Death Dis. 2017;8(10):e3153.

25. Mendell JT, Olson EN. MicroRNAs in stress signaling and human disease. Cell. 2012;148(6):1172-1187.

26. Inui M, Martello G, Piccolo S. MicroRNA control of signal transduction. Nat Rev Mol Cell Biol. 2010;11(4):252-263.

27. Mei L-L, Wang W-J, Qiu Y-T, Xie X-F, Bai J, Shi Z-Z. miR-145-5p suppresses tumor cell migration, invasion and epithelial to mesenchymal transition by regulating the Sp1/NF- $\kappa \mathrm{B}$ signaling pathway in esophageal squamous cell carcinoma. Int J Mol Sci. 2017;18(9):1833.

28. Xu W, Chang J, du X, Hou J. Long non-coding RNA PCAT-1 contributes to tumorigenesis by regulating FSCN1 via miR-145-5p in prostate cancer. Biomed Pharmacother. 2017;95:1112-1118.

29. Lu Q, Shan S, Li Y, Zhu D, Jin W, Ren T. Long noncoding RNA SNHG1 promotes non-small cell lung cancer progression by up-regulating MTDH via sponging miR-145-5p. Faseb J. 2018;32(7):3957-3967.

30. Slattery ML, Herrick JS, Mullany LE, et al. An evaluation and replication of miRNAs with disease stage and colorectal cancer-specific mortality. Int J Cancer. 2015;137(2):428-438.

31. Tavares MR, Pavan IC, Amaral CL, Meneguello L, Luchessi AD, Simabuco FM. The S6K protein family in health and disease. Life Sci. 2015;131:1-10.

32. Zhang LJ, Lu R, Song YN, et al. Knockdown of anion exchanger 2 suppressed the growth of ovarian cancer cells via mTOR/p70S6K1 signaling. Sci Rep. 2017;7(1):6362.

33. Jafari N, Zheng Q, Li L, et al. p70S6K1 (S6K1)-mediated phosphorylation regulates phosphatidylinositol 4-phosphate 5-kinase type I $\gamma$ degradation and cell invasion. J Biol Chem. 2016;291(49):25729-25741.
Cancer Management and Research

\section{Publish your work in this journal}

Cancer Management and Research is an international, peer-reviewed open access journal focusing on cancer research and the optimal use of preventative and integrated treatment interventions to achieve improved outcomes, enhanced survival and quality of life for the cancer patient. The manuscript management system is completely online and includes

\section{Dovepress}

a very quick and fair peer-review system, which is all easy to use. Visit http://www.dovepress.com/testimonials.php to read real quotes from published authors. 